\title{
Cardiopulmonary resuscitation of cardiac failure patients by using an automatic heart massage with ventilator and radio-Lucent
}

\begin{abstract}
Introduction: In the era when there are little knowledge oriented people and its propagators are often elusive of their real responsibility, we decided to, by approval the of technological research centre of Iran University of Medical Sciences, present the most complete CPR patent with a sense of responsibility towards medical society and medical culture. We hope to detect other insufficiencies in future.

Methodology: In this study, patients were brought after a cardiac failure, or were subjected to cardiac failure receiving healing operations. Success percentage of CPR group or hypoxia of is studied. Sample data is analyzed by qualitative content analysis method. This study was approved by the professors and the faculty and vice president of the Institute as well as emergency and coat lips and cardiovascular Sahid Madani. The collected data were analyzed by conventional analysis.

Findings: During this research and interviews with respected CPR and the families of victims of cardiac failure, reasons for the late arrival to clinics and lack of recovery in the transfer of patients to medical centres as well as some causes of death of some heart patients are listed. These led to propose the presence of cardiopulmonary resuscitation device with the ability of radiolucent in ambulances, revive chamber and especially in angiography part of clinics to reduce cardiac mortality.

Conclusion: Experiences gained by CPR group and ambulance group shows that Lack of sufficient awareness of families in how to deal with patients subjected to cardiac failure followed by stress and fear about trying, And delay of emergency and ambulance team and lack of on time resuscitation due to lack of manpower in ambulances as well as the absence of anesthesiologist at the moment of the patient's arrival to the emergency department are the most important causes of failure to revive some patients and may cause hypoxia. Since, the need for cardiac and pulmonary resuscitation devices in ambulances and medical centres to save the lives of these cases was reported. Radiolucent device allows subjecting the patient whom is being resuscitated by cardiopulmonary resuscitation to angiography and angioplasty to achieve the diagnosis and treatment.
\end{abstract}

Keywords: cardiopulmonary resuscitation, heart massage machine, radiolucent, CPR, ventilator
Volume II Issue 6 - 2018

\author{
Davood Momen Bahador,' Rezayat parvizi, ${ }^{2}$ \\ Abbas afrasiabi $^{3}$ \\ 'Clinical consultant and consultant of medical equipment of Talie \\ Mehr, medical and nursing care Centre, Iran \\ ${ }^{2}$ Cardiovascular surgeon, Professor of Cardio vascular, Medical \\ and research centre of Tabriz, Iran \\ ${ }^{3}$ Cardio vascular surgeon, Professor of Shahid Madani Tabriz \\ cardio vascular, medical research center of Tabriz, Iran
}

Correspondence: David Momen Bahadur, Clinical consultant and consultant of medical equipment of Talie Mehr, medical and nursing care Centre, Iran, Email davidmdahador@yahoo.com

Received: March 06, 2018 | Published: November 30, 2018

\section{Positions of device's beds}

A. For positions of machine bed that the patient is placed on, based on references of 2015, thorax parts were considered 10 to 30 degrees.

B. Scientific reason: To prevent aspiration, especially comatose patients and after 20 weeks gestation as well as the maximum systolic pressure of the patient during resuscitation by heart massage remained normal.

\section{Discussion}

A. Controversy: automotive chest compression, ventilation \& Radiolucent, Capnograph Device, A New Generation.

B. Technical evaluation of revive based on references of 2015:

C. The number of chest compressions should be100 to 120 times per minute. It means 100 to 120 Active compressions and 120 De compressions per minute.

D. Chest compression should be proportional to the chest diameter, i.e. About $4 \mathrm{~cm}$ for children and $5.4 \mathrm{~cm}$ for teens.
E. Based on the report of references of 2015 and 2016 this amount should be 5 to $6 \mathrm{~cm}$ for adults, and so, devices must be useful for the range of $4-6 \mathrm{~cm}$.

F. The force must be proportional to the diameter of the chest that based on madani hospital researches, forces must be between 5 $\mathrm{kg}$ up to $30 \mathrm{~kg}$.

G. Problem: The exact settings are not possible during the manual CPR operation and mostly are associated with the breach of chest and ribs.

H. Heart massage device operation: Operation is designed based on references of 2015 and 2016 and is able to link up with potential changes in future references.

I. The device loads 120 times per minute based on reference, i.e. 120 Active compression and 120 times De compression.

J. The force loaded on the chest was measured in relation to the diameter of the chest.

K. Patients having weights of 10 to $15 \mathrm{~kg}$ receives $10 \mathrm{~kg}$ of force and chest compression of $4 \mathrm{~cm}$. 
L. Weights of 20 to $30 \mathrm{~kg}$ receive the force of $15 \mathrm{~kg}$ and compression of $4 \mathrm{~cm}$.

M. Weights of $35 \mathrm{~kg}$ to $45 \mathrm{~kg}$ receive the force of $20 \mathrm{~kg}$ and chest compression of $5 / 4 \mathrm{~cm}$

N. Weight of 50 to $100 \mathrm{~kg} 25 \mathrm{~kg}$ sizes of 5 inches

O. Weight of 110 to $140 \mathrm{~kg}$ receive the force of $25 \mathrm{~kg}$ and the chest compression of $5 / 5 \mathrm{~cm}$

P. Weights above $150 \mathrm{~kg}$ receive the force of $30 \mathrm{~kg}$ and chest compression of $6 \mathrm{~cm}$

Q. Paying attention to these exact numbers by the device gives the $\mathrm{EF}$ of $55 \%$, i.e. Systolic pressure and pulmonary ventilation is kept at its maximum.

R. Radiolucent: Most of the patients who are not able to recover soon, receive angiography indications to at least detect syndrome type and anatomic causes.

S. Unfortunately, because of the impossibility of manual revive at the time of angiography it has not been reached and these cases have been fatal.

T. The bed of the device as well as chest compression part is nonoptically radiolucent and transparent because of special material which is invented by device inventor.

U. Angiography film and the chests of the studied samples prove this fact.

V. The radiolucent property of the bed and the chest compression part of the devices which fixes on the ending $1 / 3$ of the chest have raised the ability to move Patients having angiography indication to coat Lab and to subject them to angiography and angioplasty to possibly revive them.

W. It is even useful for stable operation with indication which based on the references include acute Myocardial infarction patients in which myocardia is less than $30 \%$ of necrosis.

$\mathrm{X}$. Patients of previous paragraph can be guided to surgery room supported by CPR device to have CABGS. ${ }^{1-3}$

\section{Solution}

1. Compression-decompression speed based on the references of 2015 must be 120 times per minute. In order to reach this goal, advanced electric air valve which is used in the pneumatic system of the device is able to open and close the air duct more than 120 times per minute.

2. That is, if the rate of 120 times per minute by PLC be used, electric valve for will be kept open for 0.25 seconds.

3. Compressed air of which the pressure depends on the force defined based on the diameter of the chest and is compressed by PLC behind the electrical valve and flows toward piston head of chest restrainer, and afterward, air electric valve remains closed for 0.25 second.

4. At this time, the air inside the pipe immediately flow into the air capsule by the system and pressure on the chest decreases quickly, and according to the elasticity of the chest, decompression occurs.
5. Feedback System: A system known as force feedback is embedded that if the chest resistance is less than defined standard for some reasons the force sensor reacts quickly and stops loading.

6. And also if the loaded force is more than chest resistance, in first $2 \mathrm{~cm}$ of compression the pressure will be unloaded.

7. At this time, the force can be selectively linked to PLC for a few seconds.

8. Radiolucent: the device bed and the chest compression part are made by special materials, that this radiolucent property is made non-optical and without any shadow this sheet also contains special features including ultra-lightweight. The bed is totally $1.5 \mathrm{~kg}$, very thin $(4 \mathrm{~mm})$ and very robust (capable of loading $\mathrm{kg} 400$ ).

9. Advantages: Due to active compression - decompression, the device is efficient in the reference of 2015.

10. According to the definitions of systolic pressure and EF laws, this device causes maximum systolic pressure, cardiac output and $\mathrm{EF}=\% 55$.

11. Ventilator: Due to lack of consciousness at CPR and semiconsciousness of victim for hours after resuscitation, Ventilator can be set in following mods, according to the respiratory rules:

12. CPR to a victim that is not intubated: using the 30 to 2 or 6 to 1 system, for example, every 30 chest compression at 120 times per minute, the device stops for 4 seconds and the patient, based on the reference, will be subjected to respiratory twice.

13. Breathing modes by ventilator: AMV for intubated victim that is being revived and the lungs have no activity.

14. CMV for victim whose lungs have no significant activity, but creating a negative pressure in the lungs can help to revive.

15. ACV for victims whose lungs may show some activities and the patient may have some consciousness is helpful for lungs.

16. Every time that the lungs have more activity, the activity of ventilator decreases. And also according to references, the patient should be separated from the ventilator in this mod.

17. The radiolucent bed and the chest compression part and lack of a metal in their molecular structure result in dependency of the victim to primary data.

18. For scrutiny, diagnosis and treatment or help the victim in order to achieve a positive result of revive, the patient can be moved to angiography and receive angioplasty.

19. In the case of acute myocardial infarction of myocardia and in the case of myocardial muscle necrosis up to $30 \%$, according to references of 2015:

20. Reviving by the device, move the victim toward the cardiac surgery room for CABGS, and if the case is trauma or accidental patient, CT, MRI, or chest angiography can be performed.

21. Bed devices: According to the reports of references of 2015, the part that Thorax and Femur are located has adjustable angle of 10 to 30 degrees relative to the horizon, and also has the ability to change angle as 30 degrees to the left. 
22. 10 to 30 degrees angle of thorax position: to avoid aspiration of comatose, and especially pregnant women over 20 weeks of pregnancy and in particular to establish maximum systolic pressure of $\mathrm{EF}=\% 55$ and the maximum ventilation.

23. 10 to 30 degrees angle of Femur position: for better of easier venous blood return toward the heart which encapsulates much of blood which due to its high elasticity for blood volume in the veins has a high capacity.

24. 30 degrees angle to left: for the victim with more than 20 weeks of pregnancy in order to increase the Inferior vena cava pressure, to move the diaphragm upward, and to increase aorta pressure, this angle is needed and compression must be loaded on the middle of the chest.

25. The device exactly fulfills the phrase "Cardio Pulmonary Resuscitation" and is able to linkup future reference changes. The device can be matched to all three revive methods:

26. Intubated method: according to the diameter of the chest, device presses the chest at 120 times per minute and non-stop for 4 to $6 \mathrm{~cm}$ ( 10 to $160 \mathrm{~kg}$ victims) and the ventilator according to reference, ventilates $\mathrm{O} 2$ and $\mathrm{CO} 2$ between the lungs and environment at 8 to 12 times per minute.

27. Non-intubated method: devices in the system of 30 or 15 to 2 , after every 30 or 15 times compression of the chest with the speed of 120 times per minute stops for 4 seconds, and in this 4 seconds according to the reference, does 2 respirations and next cycles will continue in the same way to a full recovery.

28. Method for women over 20 weeks of gestation: the device has the ability to change positions for 30 degrees to the left to reduce the pressure on the vena cava. And also according to the reference, due to the upward movement of the diaphragm of these victims, followed by increase of the pressure on aorta, exactly the middle of the chest must be compressed (In other methods, the ending $1 / 3$ of the chest is compressed).

29. Procedures and use of smart Automatic heart massage (CPR) with ventilator and radiolucent: put the victim on the device bed carefully and select the suitable positions of the bed.

30. Fix the Chest compression part of the device on the proper area of the patient chest (In pregnant women over 20 weeks of pregnancy the middle and for the rest of victims ending $1 / 3$ of the chest) by special belts in both sides of the patient which are fixed to the bed in one side and free in other side.

31. Then presses the POWER button and depending on the diameter of the chest touches one of the buttons below:

32. Button $1=5 \mathrm{Kg}$ force and compression of the chest of $4 \mathrm{Cm}$ : for victim of 10 to $15 \mathrm{~kg}$

33. Button (2) $=15 \mathrm{Kg}$ force and compression of the chest of $4 \mathrm{Cm}$ : for victim of 20 to $30 \mathrm{~kg}$
34. Button (3) $=20 \mathrm{Kg}$ force and chest compression of $5 / 4 \mathrm{Cm}$ : for victim of 35 to $45 \mathrm{~kg}$

35. Button (4) force $=25 \mathrm{Kg}$ and chest compression of $5 \mathrm{Cm}$ : for victims of 50 to $100 \mathrm{~kg}$

36. Button $(5)=30 \mathrm{Kg}$ force and chest compression of $5 / 5 \mathrm{Cm}$ : for victims of 110 to $140 \mathrm{~kg}$

37. Button (6) $=30 \mathrm{~kg}$ force chest compression of $6 \mathrm{~cm}$ : up for 140 $\mathrm{kg}$ victims

38. Button $7=$ to command the operation speed defined by the references, 100 to 120 times per minute for the reference of 2015.

39. Button $8=$ to command the method of non-stop operation for intubated victims.

40. Button $9=$ to command chest compressions rate method and system for non-intubated victim which is 30 to 2 in 2015 and 6 to 1 in 2016 that in every run and after stop ventilator provides oxygen immediately. According to the reference, the number can be given to the ventilator that is 2 in the reference of 2015. These are matched between functions of the ventilator and the Chest compression perfectly with each other by PLC programming.

41. Button $10=$ to link to a variety of forces based on the chest with a possible different resistance and anatomy

42. Button $11=$ to link to a variety of compression of the chest depth for possible changes in future references. ${ }^{3-6}$

\section{Acknowledgments}

None.

\section{Conflicts of interest}

Author declares that there is no conflicts of interest.

\section{References}

1. Robert W Neumar, Michael Shuster, Clifton W Callaway. 2015 American Heart Association for Cardio Pulmonary Resuscitation and Emergency Cardio Vascular care. Circulation. 2015;132(18):112.

2. AHA Guide lines for CPR and Emergency Cardio Vascular Current American Heart Association of 2011, 2015. 2015;132(18).

3. American Red Cross 1993. Standard First Aid stays well. Boston. 2015.

4. Cardiac Toorasic Surgery. 2015

5. Dana KA, Timothy RB, David LD, et al. Schwartz Principless of Surgery. 2015.

6. Yesha Iian. CPR for all, long man. London.1991. 